\title{
Comparison of Nonlinear Filtering Methods for Estimating the State of Charge of $\mathrm{Li}_{4} \mathrm{Ti}_{5} \mathrm{O}_{12}$ Lithium-Ion Battery
}

\author{
Jianping Gao' and Hongwen $\mathrm{He}^{2}$ \\ ${ }^{1}$ College of Vehicle \& Transportation Engineering, Henan University of Science and Technology, Luoyang 471023, China \\ ${ }^{2}$ National Engineering Laboratory for Electric Vehicles, School of Mechanical Engineering, Beijing Institute of Technology, \\ Beijing 100081, China
}

Correspondence should be addressed to Hongwen He; hwhebit@bit.edu.cn

Received 23 April 2015; Revised 28 May 2015; Accepted 8 June 2015

Academic Editor: Xiaosong $\mathrm{Hu}$

Copyright (C) $2015 \mathrm{~J}$. Gao and H. He. This is an open access article distributed under the Creative Commons Attribution License, which permits unrestricted use, distribution, and reproduction in any medium, provided the original work is properly cited.

\begin{abstract}
Accurate state of charge (SoC) estimation is of great significance for the lithium-ion battery to ensure its safety operation and to prevent it from overcharging or overdischarging. To achieve reliable SoC estimation for $\mathrm{Li}_{4} \mathrm{Ti}_{5} \mathrm{O}_{12}$ lithium-ion battery cell, three filtering methods have been compared and evaluated. A main contribution of this study is that a general three-step model-based battery SoC estimation scheme has been proposed. It includes the processes of battery data measurement, parametric modeling, and model-based SoC estimation. With the proposed general scheme, multiple types of model-based SoC estimators have been developed and evaluated for battery management system application. The detailed comparisons on three advanced adaptive filter techniques, which include extend Kalman filter, unscented Kalman filter, and adaptive extend Kalman filter (AEKF), have been implemented with a $\mathrm{Li}_{4} \mathrm{Ti}_{5} \mathrm{O}_{12}$ lithium-ion battery. The experimental results indicate that the proposed model-based SoC estimation approach with AEKF algorithm, which uses the covariance matching technique, performs well with good accuracy and robustness; the mean absolute error of the SoC estimation is within $1 \%$ especially with big SoC initial error.
\end{abstract}

\section{Introduction}

To address the two urgent tasks nowadays of protecting the environment and achieving energy sustainability, it is of a strategic significance on a global scale to replace the oil-dependent vehicles with electric vehicles. Lithium-ion batteries are currently considered to be the development trends of traction batteries and have been widely used in plugin hybrid electric vehicles (PHEVs) due to its high power and energy density, its high voltage, being pollution-free, having no memory effect, its long cycle life, and its low self-discharge [1-3]. Battery management system (BMS) is essential for the lithium-ion battery to maximize its performance, ensure its safety, and extend its life. Estimation for battery state of charge ( $\mathrm{SoC}$ ) is one of the most key techniques in the BMS. Nevertheless, it is difficult to accurately estimate SoC, because SoC is an inner state of each battery cell which cannot be directly measured and is greatly influenced by many factors, including ambient temperature, discharging current, and battery aging $[4,5]$. Therefore, the battery SoC has to be estimated with specific estimation techniques according to measured battery parameters, such as voltage, current, and temperature.

A wide variety of SoC estimation methods have been put forward to improve battery SoC determination, each one having its own advantage, most of which can be divided into four categories: looking-up table based methods, ampere-hour integral method, data-driven estimation methods, and model-based estimation methods [6-21]. In terms of the looking-up table based methods, the measurements of battery impedance, open circuit voltage, and so forth are commonly used to infer the SoC of batteries [69]. References $[10,11]$ put forward methods to determine battery SoC based on impedance measurements. Unfortunately, due to the uncertainty of driving cycles and the variable application environment, it is hard to measure these characteristic parameters accurately in real-time. The second type is the ampere-hour integral method, which has been widely applied to BMS and battery simulations of PHEVs [12]. The ampere-hour integral method acquires the SoC 
by integrating the current over the time. This method is simple and can be easily implemented on-board, so that it has been widely used in practice. However, as an openloop estimation algorithm, it cannot deal with problems caused by measurement noise and inaccurate initial SoC [13]. The third type is the data-driven estimation methods. Data-driven control methods merely use input-output data of the system to develop a controller. Since these methods do not require an accurate plant model, the estimations and assumptions introduced in the plant modeling step are omitted. For instance, the battery state estimations based on artificial neural network and fuzzy logic models are demonstrated with good accuracy $[14,15]$. However, these algorithms are very sensitive to their parameters and they may even be not convergent with bad parameters selection when the train data cannot completely cover the present operating conditions. The last type is model-based estimation method [16-20]. Plett used an extended Kalman filter (EKF) to identify unknown parameter and adaptively estimate the battery's SoC [16-18]. An inappropriate matrix of the system noise may lead to remarkable errors and even divergence [19]. As an alternative approach, SoC estimation via SoC-OCV look-up table is put forward and the battery open circuit voltage $(\mathrm{OCV})$ is estimated in real-time with a recursive least squares algorithm [21]. Additionally, the unscented Kalman filter (UKF) is also investigated to estimate the lithium-ion battery (LiB) SoC [20]. However, the OCV-based SoC estimation method can hardly safeguard the estimation accuracy dynamically for its open-loop characteristics.

In view of battery behavior and performance being relatively vulnerable to operating conditions and aging levels, what is important for us to do is to achieve the accurate SoC estimation in the long-term. In this point, there are three difficulties that should be considered seriously for achieving efficient and reliable battery SoC estimation for BMS: (1) accurate parametric modeling approach, (2) reliable and robust state estimation algorithm, and (3) systematic modeling and estimation scheme.

In solving the first problem, different kinds of battery models have been proposed and applied to their application field. However, if one applied them to BMS, they can hardly achieve desired performance. It is because of that the adaptive parameter update technique has been neglected; as a result, the model error will be larger as the battery aged or operated conditions changed. In dealing with the second problem, several advanced Kalman filters [13, 16-18] have been widely used and the estimation accuracies are appropriate for BMS application in a limited operating condition. For overcoming the last difficulties, the offline model and adaptive filters are widely used. However, it is limited by the unupdated battery model; the SoC estimation will be influenced by the unavoidable uncertainties from battery which aged and operating conditions varied.

The $\mathrm{Li}_{4} \mathrm{Ti}_{5} \mathrm{O}_{12}$ which can release lithium ions repeatedly for recharging and quickly for high current has been accepted as a novel anode material in $\mathrm{Li}_{4} \mathrm{Ti}_{5} \mathrm{O}_{12} \mathrm{LiB}$. Nevertheless, its dynamic behavior is very different from other LiBs. Traditional battery model fails to ensure high prediction precision in its voltage prediction. A key contribution of this study is that a general three-step model-based battery SoC estimation scheme, which includes adaptive model parameter updating technique for improving the parametric modeling performance, an open interface for employing adaptive filters to solve the hidden states from strong time-varying dynamic system and series structure based systematic modeling and estimation approach. With the proposed scheme, we have compared the performance of three commonly used filters, which include EKF algorithm-based SoC observer, UKF algorithm-based SoC observer, and AEKF algorithm-based SoC observer. In addition, the Gaussian model describing the open circuit voltage behavior has been developed to improve the performance of the battery model, and then the improved lumped-parameter battery model was applied to the three filters based SoC estimator to improve their prediction performance for $\mathrm{Li}_{4} \mathrm{Ti}_{5} \mathrm{O}_{12} \mathrm{LiB}$ (its voltage bounds are $2.7 \mathrm{~V}$ and $1.5 \mathrm{~V}$, resp.).

This paper is organized as follows. A description of the general model-based battery SoC estimation scheme is given in Section 2. The verification experiments and results are described in Section 3. A detailed comparison among the EKF-based, UKF-based, and AEKF-based SoC estimation approaches is illustrated in Section 4. Finally, conclusions are drawn in Section 5.

\section{Model-Based SoC Estimator}

2.1. General Model-Based Battery SoC Estimation Scheme. Figure 1 presents the model-based battery SoC estimation scheme. It contains three steps.

Step 1 (real-time measurement). After the driving cycles loaded on the battery, the data-sampling module can measure battery current and voltage in real-time. The measured current and voltage are served as input data for the next modeling and state estimation steps.

Step 2 (parametric modeling). Based on the previous research experience on battery model selection [21, 22], one RC network based lumped-parameter battery model (shown in Figure 2) has been chosen for describing the dynamic behavior of the LiB cell. In parametric modeling step, the battery model has been divided into two parts: OCV and dynamic voltage. Considering that OCV varies with battery SoC, it is common to use variation of OCV to achieve an accurate feedback of SoC. Therefore, we use a nonlinear battery OCV model to describe battery voltage source. With the estimated SoC, the OCV will be updated accordingly. For dynamic voltage part, we use recursive least squares with optimal forgetting factor to identify the parameter in realtime. The description of recursive least squares can be found in [22]. Through the process of Step 2, we can get online model parameter with real-time measurements of battery current and voltage.

Step 3 (model-based approach). With the real-time battery model from Step 2, we can build the state-space function of the battery accordingly. With the state-space function, the adaptive filters can be used to estimate the hidden state (SoC) 


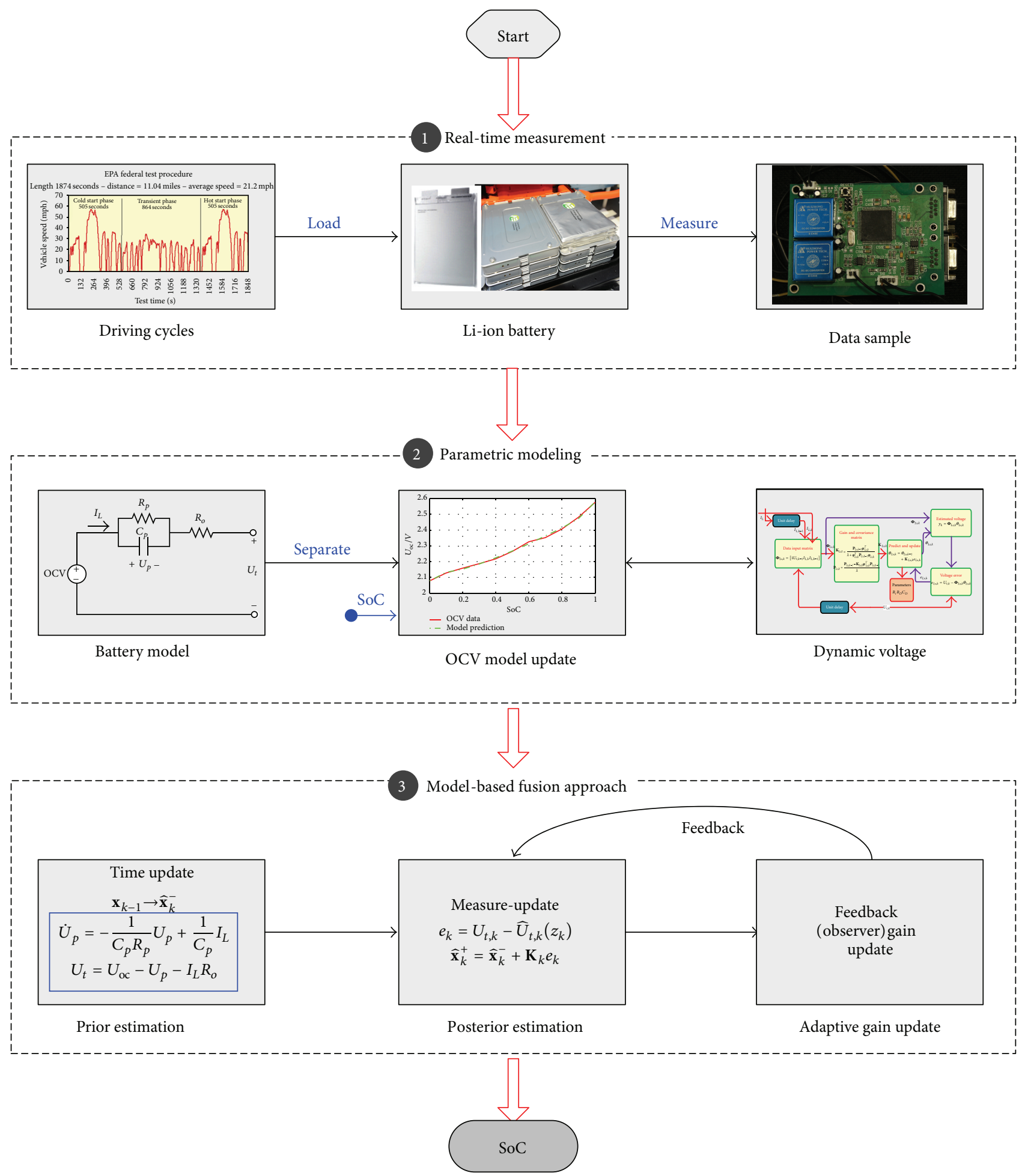

Figure 1: General model-based battery SoC estimation scheme.

through operation processes of prior estimation, posterior estimation, and feedback control. In this study, we use three kinds of Kalman filters to verify this approach [21, 23, 24]. The three kinds of advanced Kalman filters have been employed to develop the SoC estimator and their performance has been analyzed and evaluated in Section 4.
It is noted that in essence the Kalman filter based SoC estimation approach is a fusion method. It fuses the estimation results of OCV-based look-up table method and amperehour counting method through the state-space function of battery. SoC serves the communication link between OCVbased look-up table method and ampere-hour counting 


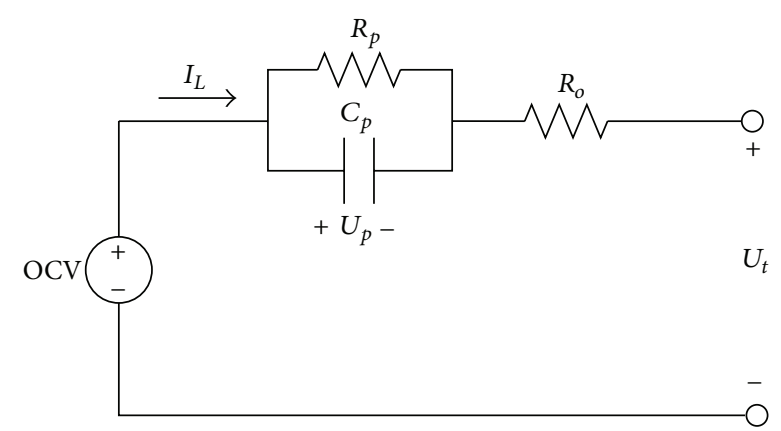

FIgURE 2: The schematic diagram of the lumped-parameter model.

method; an inaccurate ampere-hour counting method will lead to inaccurate SoC estimate and results in a larger erroneous $\mathrm{OCV}$, which will bring bigger voltage error in turn. Thus, the OCV can be served as a feedback control link to regulate the SoC estimation error in ampere-hour counting method. Therefore, the fusion method has optimal SoC estimation from its capacities of correction, weighting, and filtering. Lastly, together with the real-time measurements of battery current and voltage for online identification of the parameter of battery model, the proposed scheme in fact is model-based fusion method.

2.2. Battery Model. In order to execute the state estimation with state-space-based filter algorithm, we need a model to describe relationship between SoC with battery voltage. According to our previous experience in battery modeling [21], one RC network based lumped-parameter model was chosen for this study. However, to improve the model's accuracy and enhance the relationship between the SoC with battery voltage, a Gaussian model [17] is developed to improve its prediction precision; the model is illustrated in Figure 2. The main dynamic voltage behavior is described by

$$
\begin{aligned}
& \dot{U}_{p}=-\frac{1}{C_{p} R_{p}} U_{p}+\frac{1}{C_{p}} I_{L}, \\
& U_{t}=U_{\mathrm{oc}}-U_{p}-I_{L} R_{o},
\end{aligned}
$$

where $U_{\mathrm{oc}}$ and $I_{L}$ are the OCV and load current, respectively. $U_{t}$ and $R_{o}$ denote the terminal voltage and ohmic resistance of battery, respectively. The RC network is employed to describe the dynamic voltage behavior including polarization resistance $R_{p}$ and polarization capacitance $C_{p}$, and $U_{p}$ represents the polarization voltage across $C_{p}$. It is noted that the positive value of battery current is assumed for discharging operation while the negative value is assumed for charging operation.

To describe the nonlinear characteristic of battery open circuit voltage, the Gaussian model has been selected:

$$
\begin{aligned}
U_{\mathrm{oc}}= & a_{1} \exp \left(-\left(\frac{\left(z-b_{1}\right)}{c_{1}}\right)^{2}\right) \\
& +a_{2} \exp \left(-\left(\frac{\left(z-b_{2}\right)}{c_{2}}\right)^{2}\right) \\
& +a_{3} \exp \left(-\left(\frac{\left(z-b_{3}\right)}{c_{3}}\right)^{2}\right),
\end{aligned}
$$

where $a_{i}, b_{i}, c_{i}(i=1,2,3)$ are the constants chosen to make the $U_{\mathrm{oc}}$ model fit the SoC-OCV well and $z$ is the abbreviation of battery SoC.

2.3. Parameters Identification. For identifying the parameters of the lumped-parameter battery model, a regression equation in discretization form for (1) is needed which has been rewritten as follows [3]:

$$
\begin{aligned}
U_{p, k+1} & =e^{-\Delta t / \tau} U_{p, k}+\left(1-e^{-\Delta t / \tau}\right) R_{p} I_{L, k}, \\
U_{t, k} & =U_{\mathrm{oc}, k}-U_{p, k}-I_{L, k} R_{o},
\end{aligned}
$$

where $\Delta t$ denotes the sampling interval and $U_{p, k+1}$ can be equivalent to $U_{p}((k+1) \Delta t)$ at the $(k+1)$ th sampling times. $\tau$ denotes the time constant and equals $\tau=R_{p} \times C_{p} . I_{L, k}$ is the current at the time index $k$, and $U_{t, k}$ and $U_{\mathrm{oc}, k}$ are terminal voltage and OCV at the time index $k$, respectively.

With (3),

$$
\begin{aligned}
U_{t, k+1}= & U_{\mathrm{oc}, k+1}-\left(e^{-\Delta t / \tau} U_{p, k}+\left(1-e^{-\Delta t / \tau}\right) R_{p} I_{L, k}\right) \\
& -I_{L, k+1} R_{o} .
\end{aligned}
$$

Defining $E_{t}=U_{t}-U_{\mathrm{oc}}$, then (4) can be rewritten by

$$
\begin{aligned}
E_{t, k+1}= & -\left(e^{-\Delta t / \tau} U_{p, k}+\left(1-e^{-\Delta t / \tau}\right) R_{p} I_{L, k}\right) \\
& -I_{L, k+1} R_{o} .
\end{aligned}
$$

The difference equation of (5) can be used to eliminate $U_{p, k}$; then,

$$
\begin{aligned}
E_{t, k+1}= & e^{-\Delta t / \tau} E_{t, k}+\left(-R_{o}\right) I_{L, k+1} \\
& +\left(e^{-\Delta t / \tau} R_{o}-\left(1-e^{-\Delta t / \tau}\right) R_{p}\right) I_{L, k} .
\end{aligned}
$$

Thus,

$$
E_{t, k+1}=\alpha_{1} E_{t, k}+\alpha_{2} I_{L, k+1}+\alpha_{3} I_{L, k}
$$

where

$$
\begin{aligned}
& \alpha_{1}=e^{-\Delta t / \tau}, \\
& \alpha_{2}=-R_{o}, \\
& \alpha_{3}=e^{-\Delta t / \tau} R_{i}-\left(1-e^{-\Delta t / \tau}\right) R_{p} .
\end{aligned}
$$

From (8), we can obtain

$$
\begin{aligned}
R_{i} & =-\alpha_{2} \\
R_{p} & =\frac{\alpha_{1} \alpha_{2}+\alpha_{3}}{\alpha_{1}-1}, \\
C_{p} & =\frac{\left(1-\alpha_{1}\right) \Delta t}{\left(\alpha_{1} \alpha_{2}+\alpha_{3}\right) \log \left(\alpha_{1}\right)} .
\end{aligned}
$$

We assume $y_{k}=\boldsymbol{\Phi}_{L s, k} \boldsymbol{\theta}_{L s, k}$, where

$$
\begin{aligned}
y_{k} & =E_{t, k}, \\
\boldsymbol{\Phi}_{L s, k} & =\left[\begin{array}{lll}
U_{t, k-1} & I_{L, k} & I_{L, k-1}
\end{array}\right], \\
\boldsymbol{\theta}_{L s, k} & =\left[\begin{array}{lll}
\alpha_{1} & \alpha_{2} & \alpha_{3}
\end{array}\right]^{T},
\end{aligned}
$$


where $\boldsymbol{\Phi}_{L s, k}$ and $\boldsymbol{\theta}_{L s, k}$ denote matrixes of input data and parameters at time index $k$, respectively.

We use the recursive least squares to implement the parameters estimating process; the parameter estimates are updated at each sampling intervals. The forgetting factor $\lambda$ is optimized by the genetic algorithm and the optimization objective is to achieve the minimum sum of squares of the estimated voltage error. After presetting initial vector $\boldsymbol{\theta}_{L s, 0}$ and its error covariance matrix $\mathbf{P}_{L s, 0}$, the parameter vector $\boldsymbol{\theta}_{L s, k}$ can be determined.

2.4. SoC Estimation with the Kalman Filter Algorithm. As mentioned in the introduction section, SoC is a necessary index for ensuring the safety operation of the batteries. SoC can be calculated by the following equation:

$$
z_{k}=z_{k-1}-\frac{\eta_{i} I_{L, k} \Delta t}{C_{a}},
$$

where $z_{k}$ denotes the SoC at the $k$ th sampling interval and $\eta_{i}$ denotes the columbic efficiency of battery. $\Delta t$ is the sampling interval. $C_{a}$ denotes the maximum available capacity of battery.

Based on the dynamic voltage model of battery and the SoC computational equation, we can build the state equation for recursive prediction and the state equation is described by the following equation:

$$
\begin{gathered}
\mathbf{X}_{k+1}=f\left(\mathbf{X}_{k}, \mathbf{u}_{k}\right)+\omega_{k}, \\
\mathbf{Y}_{k}=h\left(\mathbf{X}_{k}, \mathbf{u}_{k}\right)+v_{k},
\end{gathered}
$$

where the function $f(X, u)$ is used to describe the state transition of the nonlinear system, the parameter $\mathbf{X}$ denotes the system state, and the $\mathbf{u}$ denotes the input of this system. The function $g(X, u)$ is used to describe the measurement process of this nonlinear system, $Y$ denotes the measurement of the system. To describe the unavoidable noise information, a Gaussian white noise was assumed to describe the process noise (w) and measurement noise (v). These two types of noise possess zero of mean values, and their covariance is described by vectors $\mathbf{Q}$ and $\mathbf{R}$, respectively.

A few of nonlinear filtering methods have been applied to determining the SoC for electric vehicles batteries, especially of Kalman filtering methods. They are extensively used not only for parameter identification and stats estimation, but also for other typical engineering problems such as global positioning system $[16-18,21]$. Based on [16-18], we can conclude the detailed computational process for EKF algorithm and AEKF algorithm, which have been listed below. And the algorithm of UKF algorithm can be found in [13].

Summary of the EKF algorithm is as follows.

Step 1 (initialization). For $k=0$, set

$$
\begin{aligned}
& \widehat{\mathbf{X}}_{0}^{+}=E\left[X_{0}\right] \\
& \mathbf{P}_{0}^{+}=E\left[\left(X_{0}-\widehat{\mathbf{X}}_{0}^{+}\right)\left(X_{0}-\widehat{\mathbf{X}}_{0}^{+}\right)^{T}\right] .
\end{aligned}
$$

Step 2 (computation). For $k=1,2, \ldots$, compute the following.

State estimate time update is as follows:

$$
\widehat{\mathbf{X}}_{k}^{-}=f\left(\widehat{\mathbf{X}}_{k-1}^{+}, \mathbf{u}_{k}\right) \text {. }
$$

Error innovation is as follows:

$$
\mathbf{e}_{k}=\mathbf{Y}_{k}-g\left(\widehat{\mathbf{X}}_{k}^{-}, \mathbf{u}_{k}\right)
$$

Error covariance time update is as follows:

$$
\mathbf{P}_{k}^{-}=\mathbf{A}_{k} \mathbf{P}_{k-1} \mathbf{A}_{k}^{T}+\mathbf{Q}_{k}
$$

Kalman gain matrix is as follows:

$$
\mathbf{K}_{k}=\mathbf{P}_{k}^{-} \mathbf{C}_{k}^{T}\left(\mathbf{C}_{k} \mathbf{P}_{k}^{-} \mathbf{C}_{k}^{T}+\mathbf{R}_{k}\right)^{-1} .
$$

State estimate measurement update is as follows:

$$
\widehat{\mathbf{X}}_{k}^{+}=\widehat{\mathbf{X}}_{k}^{-}+\mathbf{K}_{k} \mathbf{e}_{k}
$$

Noise and error covariance measurement update is as follows:

$$
\mathbf{P}_{k}^{+}=\left(\mathbf{I}-\mathbf{K}_{k} \mathbf{C}_{k}\right) \mathbf{P}_{k}^{-},
$$

where

$$
\begin{aligned}
& \mathbf{A}_{k}=\left.\frac{\partial f\left(\mathbf{X}_{k}, \mathbf{u}_{k}\right)}{\partial \mathbf{X}}\right|_{\mathbf{X}=\widehat{\mathbf{X}}_{k}^{-}}, \\
& \mathbf{C}_{k}=\left.\frac{\partial h\left(\mathbf{X}_{k}, \mathbf{u}_{k}\right)}{\partial \mathbf{X}}\right|_{\mathbf{X}=\widehat{\mathbf{X}}_{k}^{-}},
\end{aligned}
$$

where $\mathbf{K}_{k}$ is Kalman gain matrix at the $k$ th sampling time; $\widehat{\mathbf{X}}_{k}^{-}$ is the priori estimate of $\mathbf{X}_{k}$ before the measurement $\mathbf{Y}_{k}$ is taken into account, $\widehat{\mathbf{X}}_{k}^{+}$is the estimate of $\mathbf{X}_{k}$ after the measurement $\mathbf{Y}_{k}$ is taken into account, which is called posteriori estimate; $U_{t, k}$ and $\widehat{U}_{t, k}$ are the terminal voltage measured and estimated by the battery model at the $k$ th sampling time, respectively.

Summary of the AEKF algorithm is as follows.

Step 1 (initialization). For $k=0$, set:

$$
\begin{aligned}
& \widehat{\mathbf{X}}_{0}^{+}=E\left[X_{0}\right], \\
& \mathbf{P}_{0}^{+}=E\left[\left(X_{0}-\widehat{\mathbf{X}}_{0}^{+}\right)\left(X_{0}-\widehat{\mathbf{X}}_{0}^{+}\right)^{T}\right] .
\end{aligned}
$$

Step 2 (computation). For $k=1,2, \ldots$, compute the following.

State estimate time update is as follows:

$$
\widehat{\mathbf{X}}_{k}^{-}=f\left(\widehat{\mathbf{X}}_{k-1}^{+}, \mathbf{u}_{k}\right) .
$$

Error innovation is as follows:

$$
\mathbf{e}_{k}=\mathbf{Y}_{k}-g\left(\widehat{\mathbf{X}}_{k}^{-}, \mathbf{u}_{k}\right) .
$$


TABLE 1: Results of the coulomb efficiency.

\begin{tabular}{lccccc}
\hline Current/A & 3 & 10 & 20 & 40 & 60 \\
\hline Coulombic efficiency in discharging process & 1 & 1 & 0.99 & 0.98 & 0.95 \\
Coulombic efficiency in charging process & 1 & 1 & 0.99 & 0.97 & - \\
\hline
\end{tabular}

Adaptive law-covariance matching is as follows:

$$
\begin{aligned}
& \mathbf{H}_{k}=\frac{1}{M} \sum_{i=k-M+1}^{k} \mathbf{e}_{k} \mathbf{e}_{k}^{T}, \\
& \mathbf{R}_{k}=\mathbf{H}_{k}-\mathbf{C}_{k} \mathbf{P}_{k}^{-} \mathbf{C}_{k}^{T} .
\end{aligned}
$$

Error covariance time update is as follows:

$$
\mathbf{P}_{k}^{-}=\mathbf{A}_{k} \mathbf{P}_{k-1} \mathbf{A}_{k}^{T}+\mathbf{Q}_{k}
$$

Kalman gain matrix is as follows:

$$
\mathbf{K}_{k}=\mathbf{P}_{k}^{-} \mathbf{C}_{k}^{T}\left(\mathbf{C}_{k} \mathbf{P}_{k}^{-} \mathbf{C}_{k}^{T}+\mathbf{R}_{k}\right)^{-1}
$$

State estimate measurement update is as follows:

$$
\widehat{\mathbf{X}}_{k}^{+}=\widehat{\mathbf{X}}_{k}^{-}+\mathbf{K}_{k} \mathbf{e}_{k}
$$

Noise and error covariance measurement update is as follows:

$$
\begin{aligned}
& \mathbf{Q}_{k}=\mathbf{K}_{k} \mathbf{H}_{k} \mathbf{K}_{k}^{T}, \\
& \mathbf{P}_{k}^{+}=\left(\mathbf{I}-\mathbf{K}_{k} \mathbf{C}_{k}\right) \mathbf{P}_{k}^{-},
\end{aligned}
$$

where $\mathbf{H}_{k}$ is the innovation covariance matrix based on the innovation sequence $\mathbf{e}_{k}$ inside moving estimation window of size $M$ at the $k$ th sampling time.

\section{Experimental Configurations and Battery Test}

A test platform introduced in our previous work [21] was employed to implement the systematic test for evaluating the performance of the proposed model-based SoC estimation algorithm. Battery used in the test is the $\mathrm{Li}_{4} \mathrm{Ti}_{5} \mathrm{O}_{12}$ lithiumion battery. The battery was placed in a thermal chamber and the test schedule can be found in [21]. The maximum available capacity of the battery is $9.8 \mathrm{Ah} \mathrm{[25].} \mathrm{After} \mathrm{the} \mathrm{static} \mathrm{capacity}$ test, an efficiency test has been implemented for determining the coulomb efficiency of the $\mathrm{Li}_{4} \mathrm{Ti}_{5} \mathrm{O}_{12}$; the results are listed in Table 1 [21].

When we know the exact capacity and coulomb efficiency, we can carry out the OCV test to calibrate the relationship between battery SoC and OCV, and we use $3 \mathrm{~A}$ to charge and discharge the cell. In this paper, the hysteresis is ignored. The OCV curves are plotted in Figure 3.

Figure 3 gives the OCV comparison between the model estimates and the test data. It suggests that the proposed

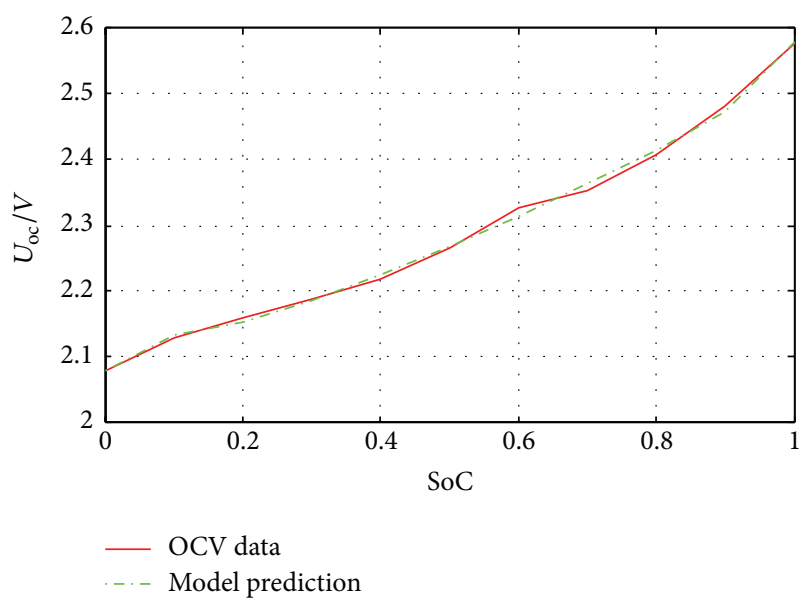

FIGURE 3: The OCV curves of the test data and the model prediction.

model can track the battery OCV behavior closely. The OCV function can be described by the following equation:

$$
\begin{aligned}
U_{\mathrm{oc}}= & 2.85 \exp \left(-\left(\frac{(z-1.614)}{1.78}\right)^{2}\right) \\
& +0.69 \exp \left(-\left(\frac{(z-0.027)}{0.56}\right)^{2}\right) \\
& +0.28 \exp \left(-\left(\frac{(z-0.071)}{0.025}\right)^{2}\right) .
\end{aligned}
$$

In addition to the above three types of test, the hybrid pulse power characteristic (HPPC) [26] and the dynamic stress test (DST) have been carried out for verifying the proposed general model-based SoC estimation method. The measurements of HPPC test and DST are plotted in Figures 4 and 5 , respectively.

To achieve an exact SoC, we first charged the battery with CCCV charge mode at the nominal current. Then we discharged some capacity of the battery with the nominal current to achieve an accurate initial SoC. Afterwards, the DST test was loaded and executed. Lastly, a further nominal current discharge experiment was conduct to gain an accurate terminal SoC. Based on the known exact SoC and accurate coulomb efficiency, we can determine the reference SoC profiles with the SoC definition-based ampere-hour counting method. The SoC profiles of DST test shown in Figure 5(c) will serve as a reference SoC for the evaluation the robust SoC estimation, and its actual initial SoC is 0.8075 . 


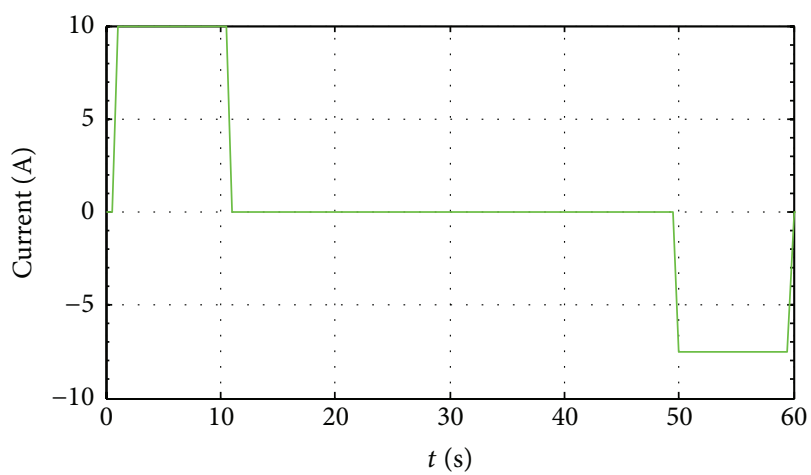

(a)

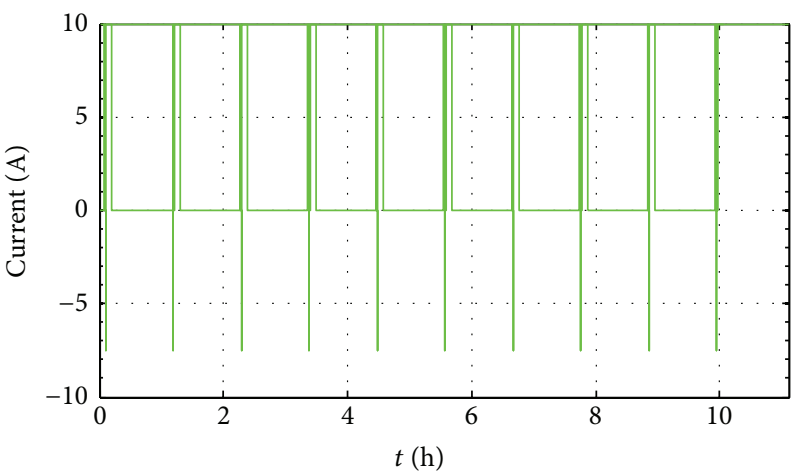

(c)

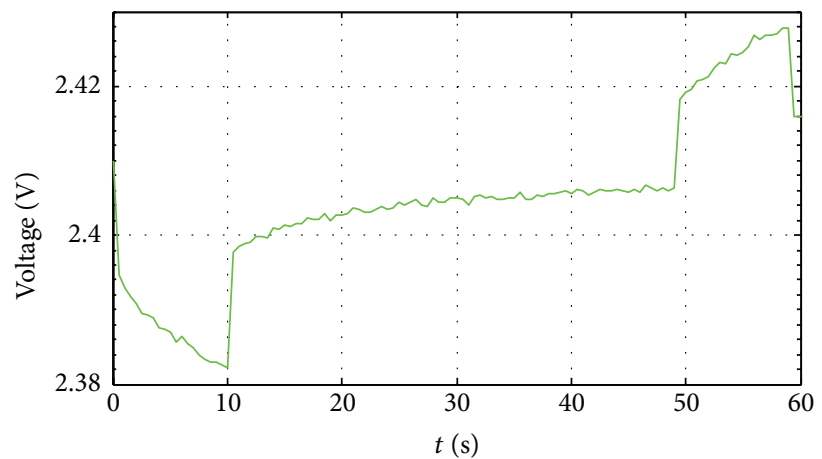

(b)

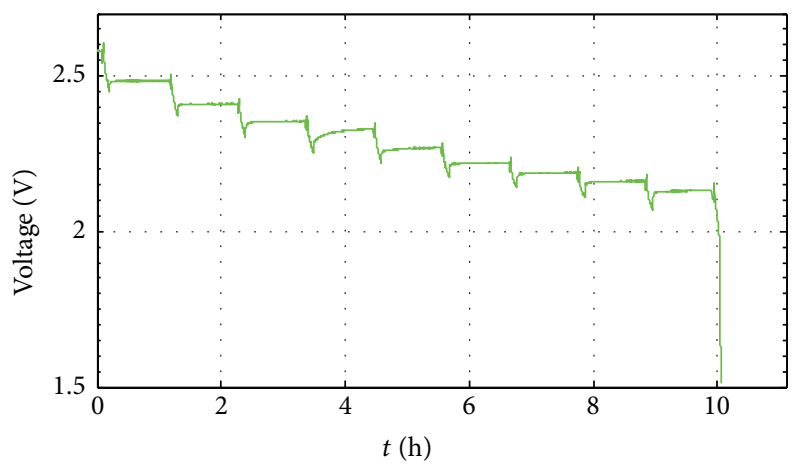

(d)

FIGURE 4: Profiles of the HPPC test: (a) battery current of one cycle; (b) battery terminal voltage (SoC = 0.8); (c) battery current for ten cycles; and (d) complete voltage profile.

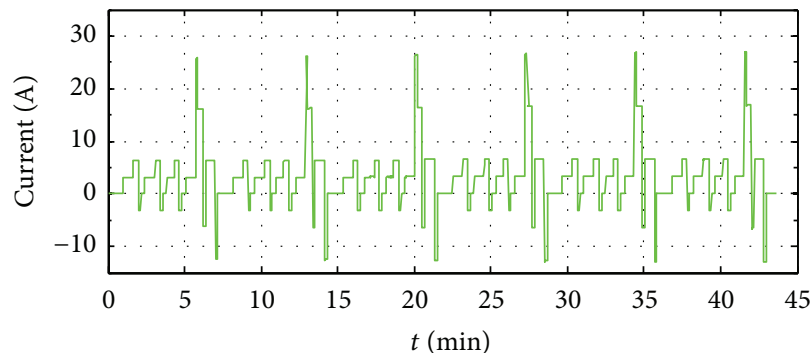

(a)

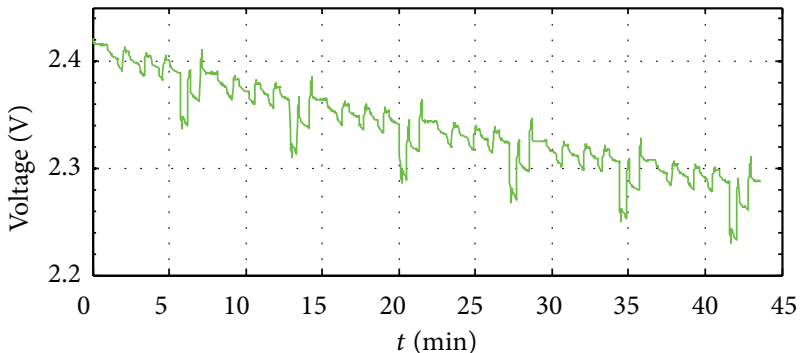

(b)

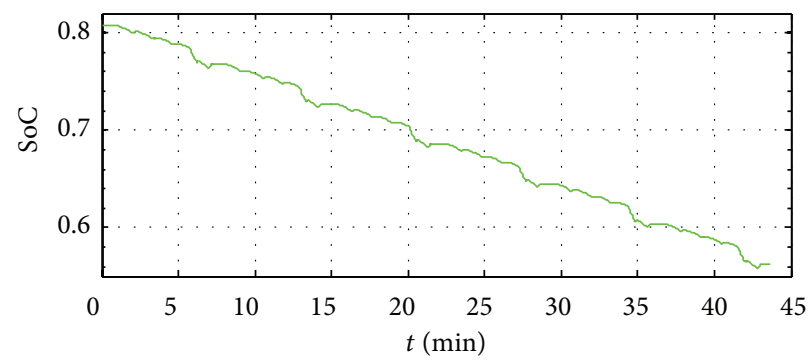

(c)

FIGURe 5: Profiles of the DST cycles: current and voltage are plotted in (a) and (b); SoC is plotted in (c). 


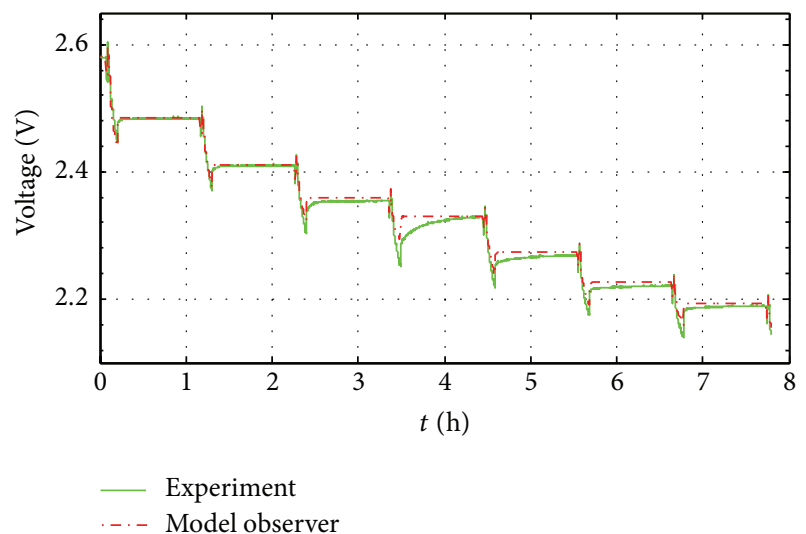

(a)

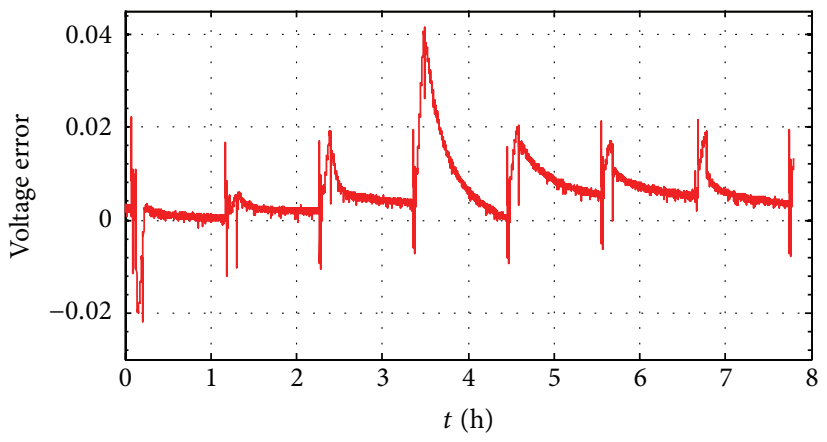

(b)

FiguRE 6: The comparisons profiles of the observer and experiment: (a) voltage; (b) voltage error.

\section{Verification Analysis and Discussion}

4.1. Parametric Modeling. Through the online parameter identification operation, we can get the real-time battery model. The voltage profiles of the experimental data and observer are presented in Figure 6(a), and the voltage error is shown in Figure 6(b). A statistical analysis on the terminal voltage errors has been conducted and the result is listed in Table 2. It shows that the maximum error of the terminal voltage is less than $2 \%$ of its nominal voltage. Additionally, its mean error is $6 \mathrm{mV}$. It can be concluded that the proposed improved model has good dynamic voltage prediction performance.

4.2. Analysis on the SoC Estimation Results. The following verification and analysis are based on the AEKF algorithm, and the other two Kalman filters will be discussed in the Section 4.3. The SoC estimation is conducted and the results are shown in Figure 8. Figure 7(a) presents the comparison profiles of the voltages between the estimations and the experimental result. Figure 7(b) presents the voltage error curve for Figure 7(a). Figure 7(c) presents the SoC comparison profiles between the estimations and the reference SoC. Figure 7(d) presents the SoC estimation error.

We can observe that the prediction inaccuracy of the battery terminal voltage is below $1 \%$, which is lower than the prediction result plotted in Figure 6(b). The prediction precision depends adaptively on correction performance by adjusting the Kalman filter gain matrix based on the voltage error bound. Figure $7(\mathrm{~d})$ indicates that the SoC estimation error arises with the terminal voltage estimation error, while the AEKF approach can correct the voltage error adaptively and quickly. It also shows that the model precision is important during the estimation for battery SoC.

4.3. Evaluation on the Robust Performance. With an accurate initial SoC, most of the SoC estimators can achieve desired estimation performance in a period of time, such as amperehour integral method. However, the estimation accuracy against different unknown initial SoC makes lots of methods
TABLE 2: The statistics list of the terminal voltage error.

\begin{tabular}{lcccc}
\hline Error & Maximum/V & Minimum/V & Mean $/ \mathrm{V}$ & Covariance $/ \mathrm{V}^{2}$ \\
\hline Value & 0.0416 & -0.0218 & 0.0060 & $3.88 e-005$ \\
\hline
\end{tabular}

unacceptable for electric vehicles application. In this section, we will discuss whether the AEKF-based SoC estimation can achieve accurate SoC estimation with the erroneous initial SoC. Two types of erroneous initial SoC, 0.95 and 0.50 , are applied to implement the evaluation. The estimation results are plotted in Figure 8.

From Figures 8(a) and 8(c), we can observe that the performance of the model-based SoC estimator cannot be affected by the erroneous initial SoC. In contrast, the convergence trajectories of the SoC estimations are very similar, especially when the initial error in battery SoC has been corrected. We also can observe that the SoC estimation errors are less than $1 \%$. In order to evaluate the estimation performance more intuitively, we select the index of the mean absolute error (MAE) to describe the convergence trajectory. The MAE can be calculated by the following equation:

$$
z_{\mathrm{mae}, k}=\frac{\sum_{j=0}^{k}\left|\mathrm{SoC}_{k}-\widehat{\mathrm{SoC}}_{k}\right|}{k+1}
$$

where $z_{\text {mae, } k}$ represents the MAE index of the battery SoC estimations up to and involving the $k$ th sample time; $\widehat{S O C}_{k}$ denotes the SoC estimation value under the time index $k$.

Figure 9 shows the MAE results. It indicates that the MAE of the SoC estimations which started with incorrect initial SoC values can converge to within $1 \%$ in 1 minute (60 sample intervals).

4.4. Discussion. Based on the above analysis, we can find that the AEKF algorithm is suitable for applying to proposed general model-based battery SoC estimation scheme, and it can reach high estimation accuracy. To discuss the suitability of the proposed general SoC estimation scheme and compare the AEKF algorithm with other widely used methods, UKF 


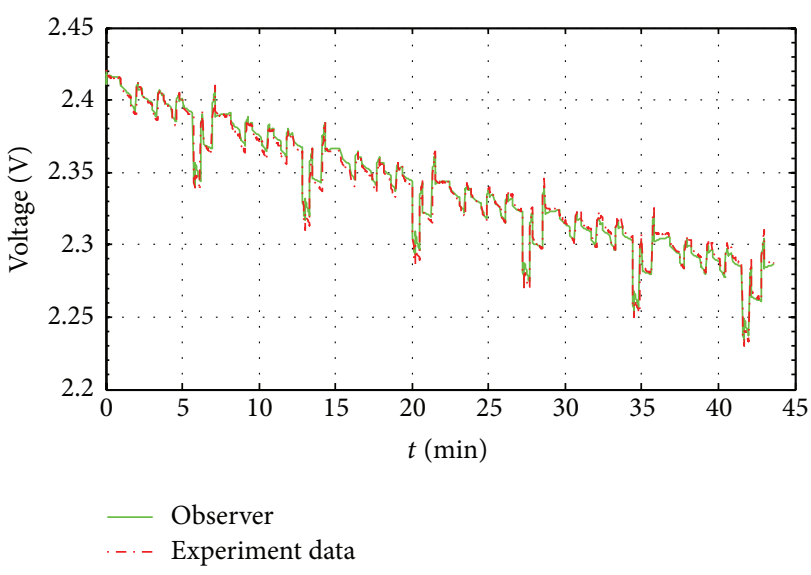

(a)

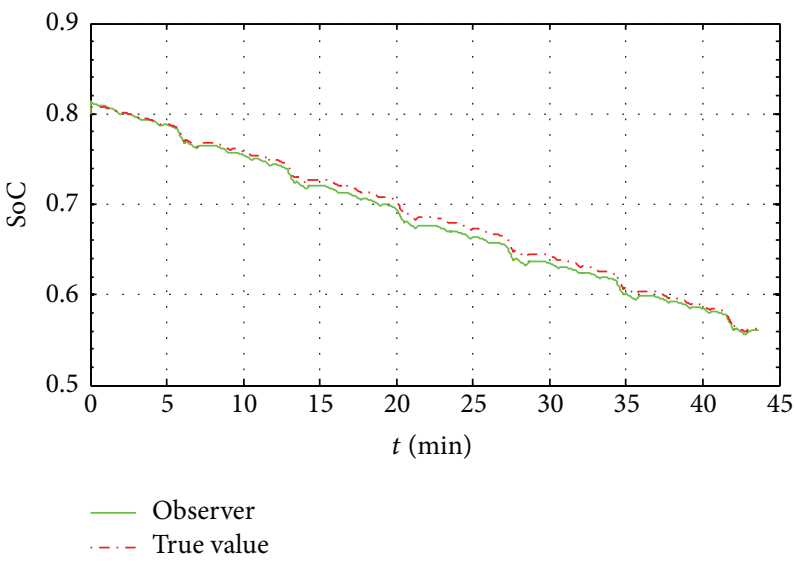

(c)

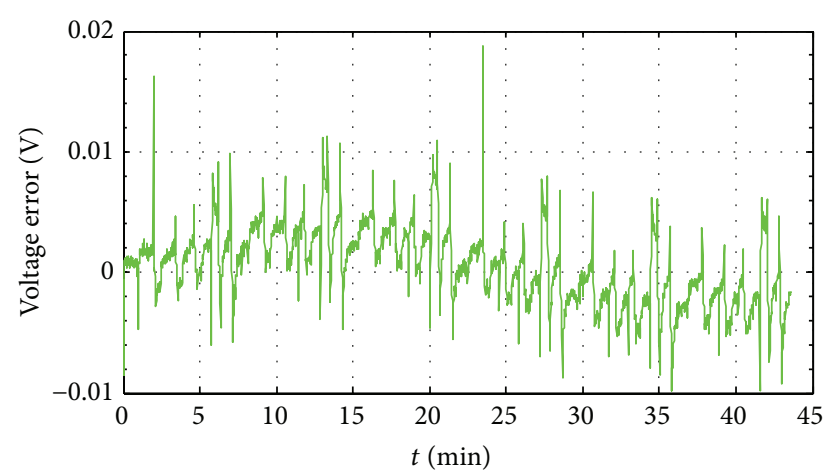

(b)

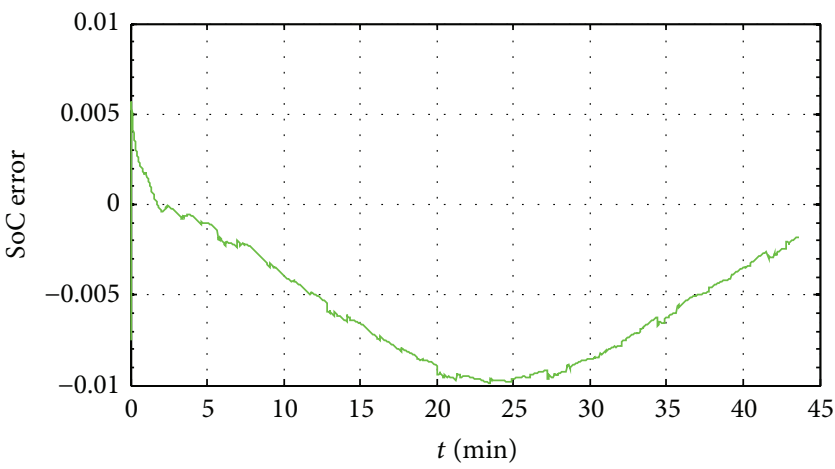

(d)

FIGURE 7: SoC estimation with AEKF approach: (a) voltage; (b) voltage error; (c) SoC; (d) SoC error.

algorithm and EKF algorithm, we have made systematical analysis. The real-time model-based SoC estimation results using the EKF and UKF are plotted in Figure 10. The estimation errors between the EKF-based SoC estimations and UKF-based estimations are plotted in Figure 11 and the MAE index is plotted in Figure 12.

From Figures 8-12, it can be observed that the AEKFbased approach achieves the best accuracy in these three approaches. Additionally, the AEKF-based SoC estimation is more precise than the EKF-based SoC estimation. The better estimation performance of the AEKF algorithm based method is due to the fact that the adaptively updating for the error covariance greatly improves the estimation performance. The SoC estimation error between the EKF-based method and the UKF-based method is virtually indiscernible.

The comparisons between the estimation inaccuracies and MAE index of the AEKF-based, EKF-based and UKFbased methods, show that the maximum MAE of the EKFbased and UKF-based approaches are around 3\%, which is higher than the AEKF-based approach. In conclusion, the proposed general model-based battery SoC estimation scheme can be applied to estimate the SoC of batteries accurately with good robust performance. More importantly, the performance of the proposed scheme is not sensitive with the operated nonlinear filtering methods. For the algorithms of AEKF, EKF, and UKF, all of their estimation errors are less than $5 \%$. It is acceptable for the current requirements of the battery management system. Furthermore, the AEKF algorithm, which can update the error covariance matrix adaptively, has the best estimation accuracy when applied to the proposed scheme and $\mathrm{Li}_{4} \mathrm{Ti}_{5} \mathrm{O}_{12}$ lithium-ion battery cell.

\section{Conclusions}

This paper presents a comparison of nonlinear filtering methods for estimating the SoC of $\mathrm{Li}_{4} \mathrm{Ti}_{5} \mathrm{O}_{12}$ lithium-ion battery. The Gaussian model has been selected to improve the prediction precision of the dynamic battery model. With the new battery model, general model-based battery SoC estimation has been proposed. It contains the adaptive model parameter updating technique for improving the parametric modeling performance, an open interface for employing adaptive filters to solve the hidden states from strong timevarying dynamic system, and series structure based systematic modeling and estimation approach. Three Kalman filters are employed to build model-based SoC estimator. With 

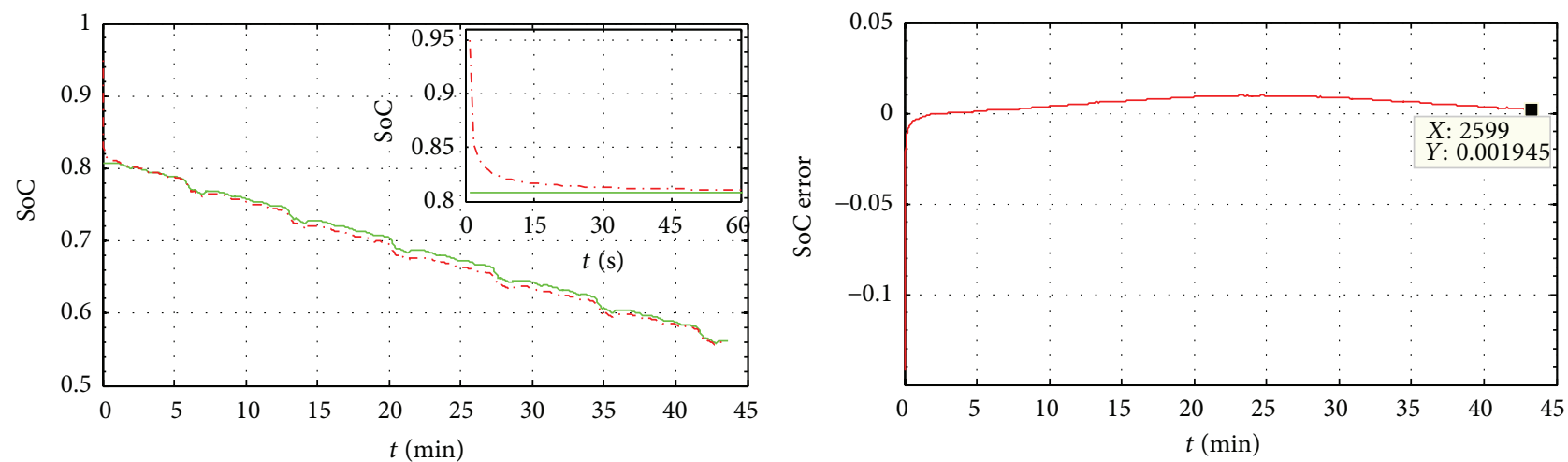

- True SoC

... AEKF

(a)
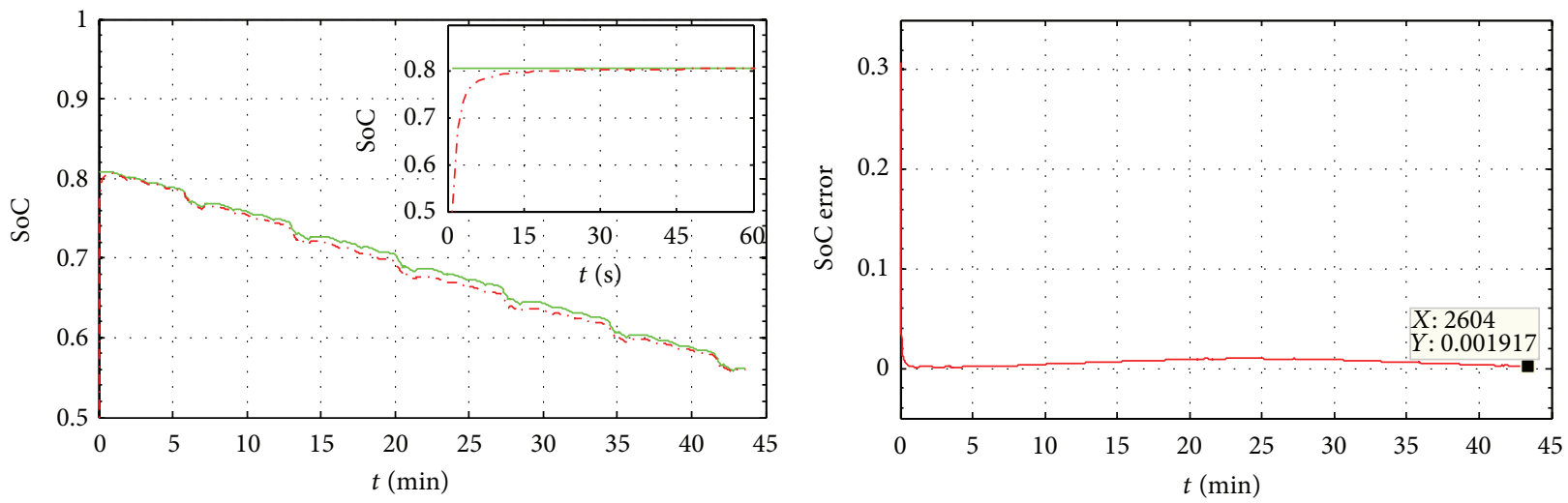

- True SoC

... AEKF

(c)

(d)

FIGURE 8: Self-correcting capability for erroneous initial SoC: (a) estimation with SoC $_{0}=0.95$; (b) SoC estimation error for (a); (c) estimation with $\mathrm{SoC}_{0}=0.50$; and (d) SoC estimation error for (c).

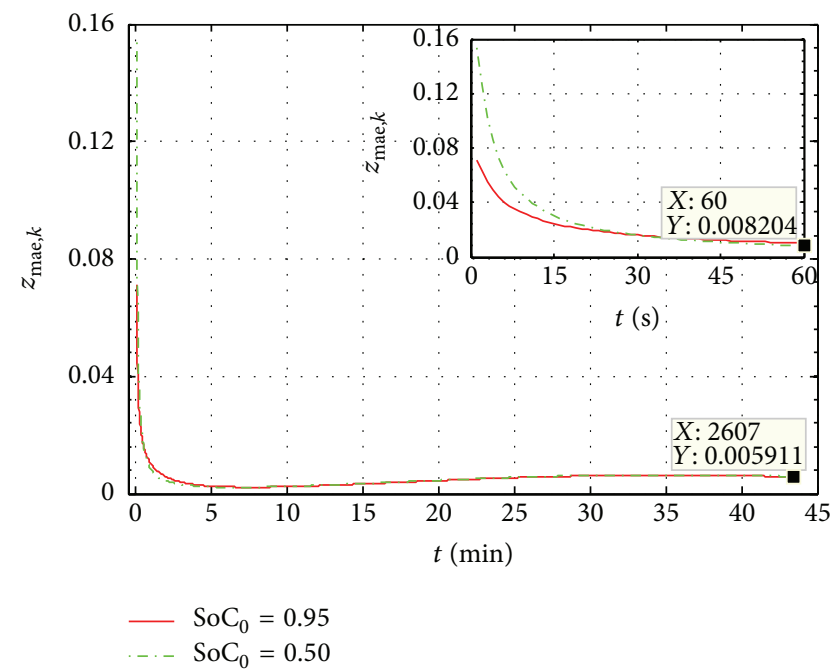

FIGURE 9: MAE results of SoC estimation with AEKF approach.

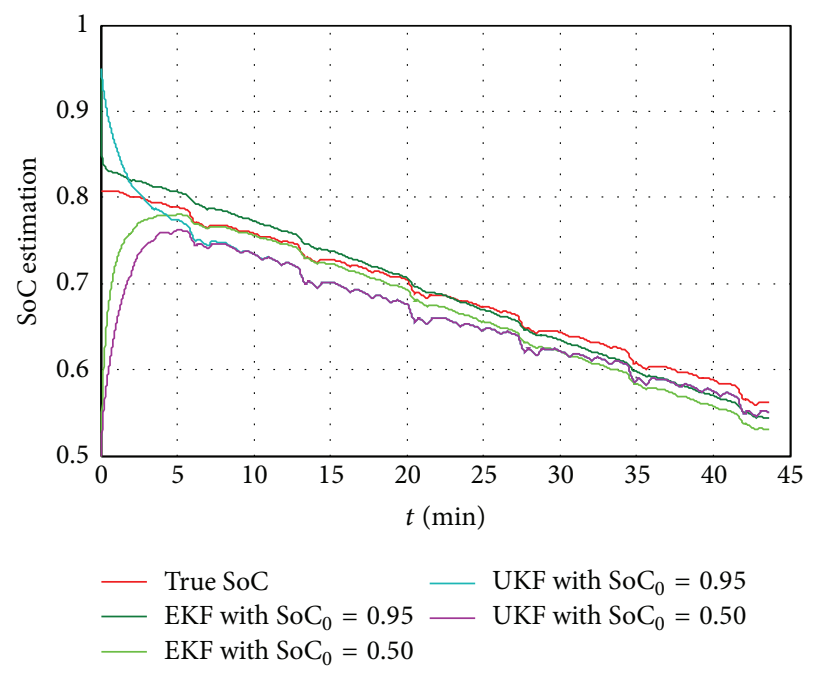

FIGURE 10: SoC results of EKF-based estimation and UKF-based estimation with two different types of $\mathrm{SoC}_{0}$. 


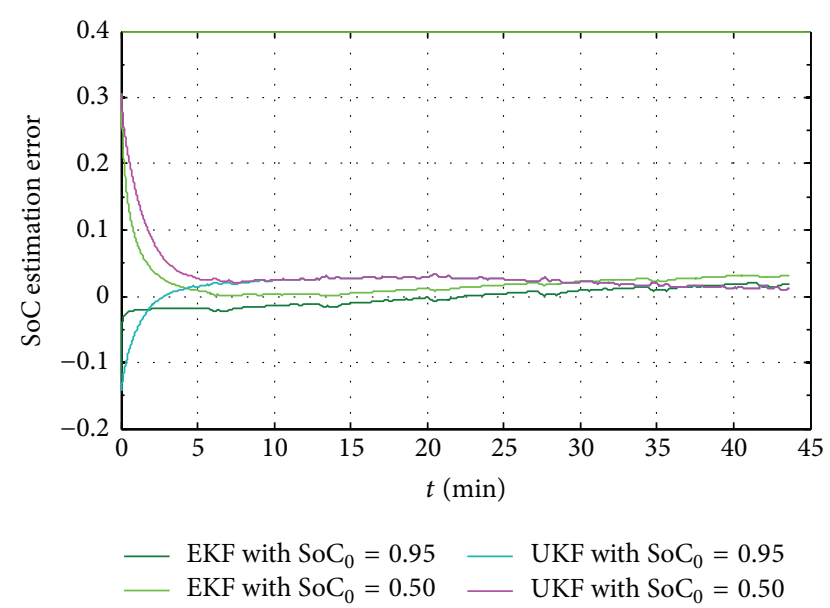

FIGURE 11: Errors of EKF-based estimation and UKF-based estimation.

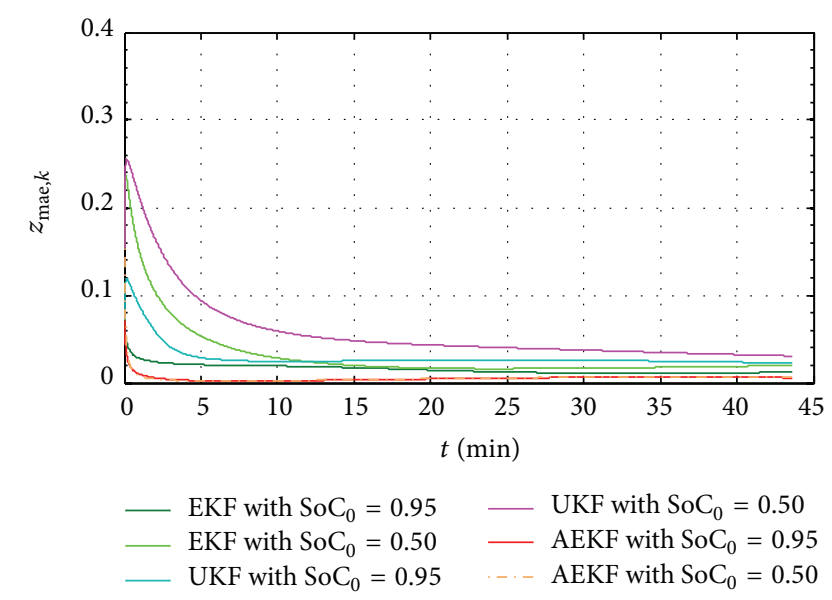

FIGURE 12: MAE results of EKF-based estimation and UKF-based estimation.

the proposed model-based scheme, three advanced Kalman filters, which include extended Kalman filter, unscented Kalman filter, and adaptive extended Kalman filter, have been employed to develop the SoC estimator.

The detailed evaluation and comparison are made for model-based SoC estimator. A comparison for the SoC estimation approach among the AEKF-based, EKF-based, and UKF-based algorithms with the $\mathrm{Li}_{4} \mathrm{Ti}_{5} \mathrm{O}_{12}$ lithiumion battery shows that the proposed method has superior performance, which indicates that the covariance matching approach for EKF is a useful way to improve its filter performance. Adaptive extended Kalman filter is an optimal choice for battery SoC estimation. Experimental results show that the AEKF-based approach can estimate the battery SoC accurately. Further, for different SoC initial values with big error, the mean absolute errors of the SoC estimation are all within 1\%; more importantly, the AEKF-based approach can ensure the estimates converge to true values quickly, less than 60 sample intervals.

\section{Conflict of Interests}

The authors declare that there is no conflict of interests regarding the publication of this paper.

\section{Acknowledgments}

This work was supported by the National Natural Science Foundation of China (51276022) and the National Science \& Technology Pillar Program (2013BAG05B00).

\section{References}

[1] J. C. Álvarez Antón, P. J. García Nieto, F. J. de Cos Juez, F. S. Lasheras, C. B. Viejo, and N. Roqueñí Gutiérrez, "Battery state-of-charge estimator using the MARS technique," IEEE Transactions on Power Electronics, vol. 28, no. 8, pp. 3798-3805, 2013.

[2] C. Blanco, L. Sanchez, M. Gonzalez, J. C. Anton, V. Garcia, and J. C. Viera, "An equivalent circuit model with variable effective capacity for $\mathrm{LiFePO}_{4}$ batteries," IEEE Transactions on Vehicular Technology, vol. 63, no. 8, pp. 3592-3599, 2014.

[3] R. Xiong, F. Sun, H. He, and T. D. Nguyen, "A data-driven adaptive state of charge and power capability joint estimator of lithium-ion polymer battery used in electric vehicles," Energy, vol. 63, pp. 295-308, 2013.

[4] Y. Xing, E. W. M. Ma, K. L. Tsui, and M. Pecht, "Battery management systems in electric and hybrid vehicles," Energies, vol. 4, no. 11, pp. 1840-1857, 2011.

[5] R. Xiong, F. Sun, Z. Chen, and H. He, "A data-driven multi-scale extended Kalman filtering based parameter and state estimation approach of lithium-ion olymer battery in electric vehicles," Applied Energy, vol. 113, pp. 463-476, 2014.

[6] C. C. Chan and K. T. Chau, Modern Electric Vehicle Technology, Oxford University Press, 2001.

[7] F. Huet, "A review of impedance measurements for determination of the state-of-charge or state-of-health of secondary batteries," Journal of Power Sources, vol. 70, no. 1, pp. 59-69, 1998.

[8] J. H. Aylor, A. Thieme, and B. W. Johnson, "A battery state-ofcharge indicator for electric wheelchairs," IEEE Transactions on Industrial Electronics, vol. 39, no. 5, pp. 398-409, 1992.

[9] C. C. Christianson and R. F. Bourke, "Battery state of charge gauge," US Patent 4595880, 1975.

[10] E. J. Dowgiallo Jr., "Method for determining battery state of charge by measuring A.C. electrical phase angle change," US Patent 3984762, 1975.

[11] E. Zaugg, "Process and apparatus for determining the state of charge of a battery," US Patent 4433295, 1982.

[12] V. Pop, H. J. Bergveld, D. Danilov, P. P. L. Regtien, and P. H. L. Notten, Battery Management Systems: Accurate State-of-Charge Indication for Battery-Powered Applications, Springer, London, UK, 2008.

[13] F. Sun, X. Hu, Y. Zou, and S. Li, "Adaptive unscented Kalman filtering for state of charge estimation of a lithium-ion battery for electric vehicles," Energy, vol. 36, no. 5, pp. 3531-3540, 2011.

[14] S. Grewal and D. A. Grant, "A novel technique for modelling the state of charge of lithium ion batteries using artificial neural networks," in Proceedings of the 23rd International Telecommunications Energy Conference, pp. 174-179, October 2001. 
[15] A. J. Salkind, C. Fennie, P. Singh, T. Atwater, and D. E. Reisner, "Determination of state-of-charge and state-of-health of batteries by fuzzy logic methodology," Journal of Power Sources, vol. 80, no. 1-2, pp. 293-300, 1999.

[16] G. L. Plett, "Extended Kalman filtering for battery management systems of LiPB-based HEV battery packs: part 1. Background," Journal of Power Sources, vol. 134, no. 2, pp. 252-261, 2004.

[17] G. L. Plett, "Extended Kalman filtering for battery management systems of LiPB-based HEV battery packs: part 2. Modeling and identification," Journal of Power Sources, vol. 134, no. 2, pp. 262276, 2004.

[18] G. L. Plett, "Extended Kalman filtering for battery management systems of LiPB-based HEV battery packs: part 3. State and parameter estimation," Journal of Power Sources, vol. 134, no. 2, pp. 277-292, 2004.

[19] A. H. Mohamed and K. P. Schwarz, "Adaptive Kalman filtering for INS/GPS," Journal of Geodesy, vol. 73, no. 4, pp. 193-203, 1999.

[20] H. Dai, X. Wei, and Z. Sun, "Design and implementation of a UKF-based SOC estimator for $\mathrm{LiMnO}_{2}$ batteries used on electric vehicles," Przeglad Elektrotechniczny, vol. 88, no. 1, pp. 57-63, 2012.

[21] R. Xiong, F. Sun, X. Gong, and C. Gao, "A data-driven based adaptive state of charge estimator of lithium-ion polymer battery used in electric vehicles," Applied Energy, vol. 113, pp. 1421-1433, 2014.

[22] F. Sun and R. Xiong, "A novel dual-scale cell state-of-charge estimation approach for series-connected battery pack used in electric vehicles," Journal of Power Sources, vol. 274, pp. 582-594, 2015.

[23] R. K. Mehra, "Approaches to adaptive filtering," IEEE Transactions on Automatic Control, vol. 17, no. 5, pp. 693-698, 1972.

[24] J. Han, D. Kim, and M. Sunwoo, "State-of-charge estimation of lead-acid batteries using an adaptive extended Kalman filter," Journal of Power Sources, vol. 188, no. 2, pp. 606-612, 2009.

[25] Technical specification of Battery Management System for Electric vehicles, http://www.catarc.org.cn/Upload/file/bzyj/PDF/ zhengqiuyijian-sc27-19.pdf.

[26] Idaho National Engineering \& Environmental Laboratory, Battery Test Manual for Plug-In Hybrid Electric Vehicles, Assistant Secretary for Energy Efficiency and Renewable Energy (EE) Idaho Operations Office, Idaho Falls, Idaho, USA, 2010. 


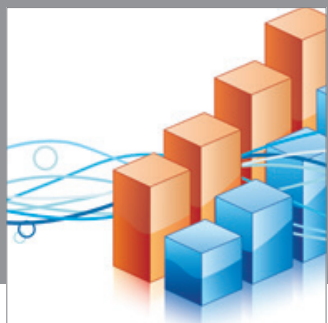

Advances in

Operations Research

mansans

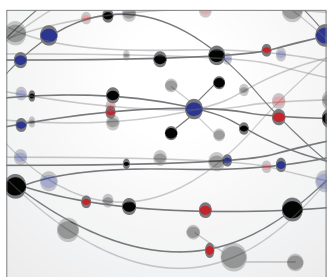

The Scientific World Journal
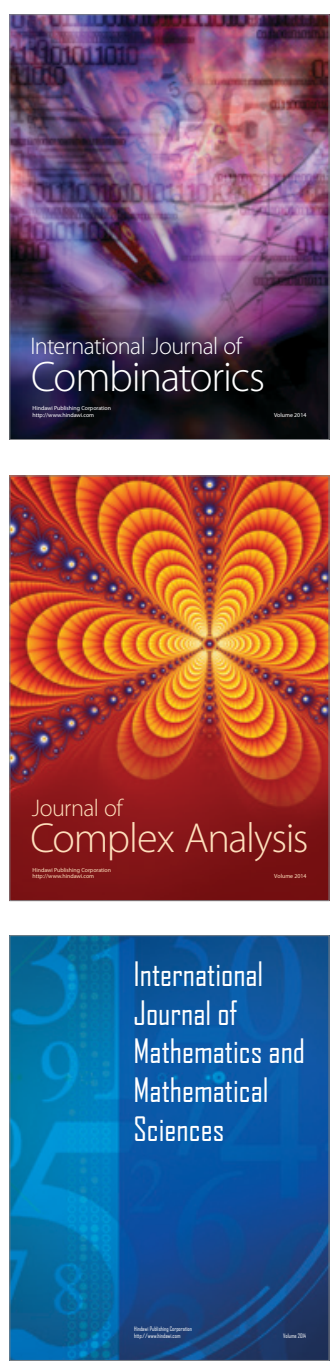
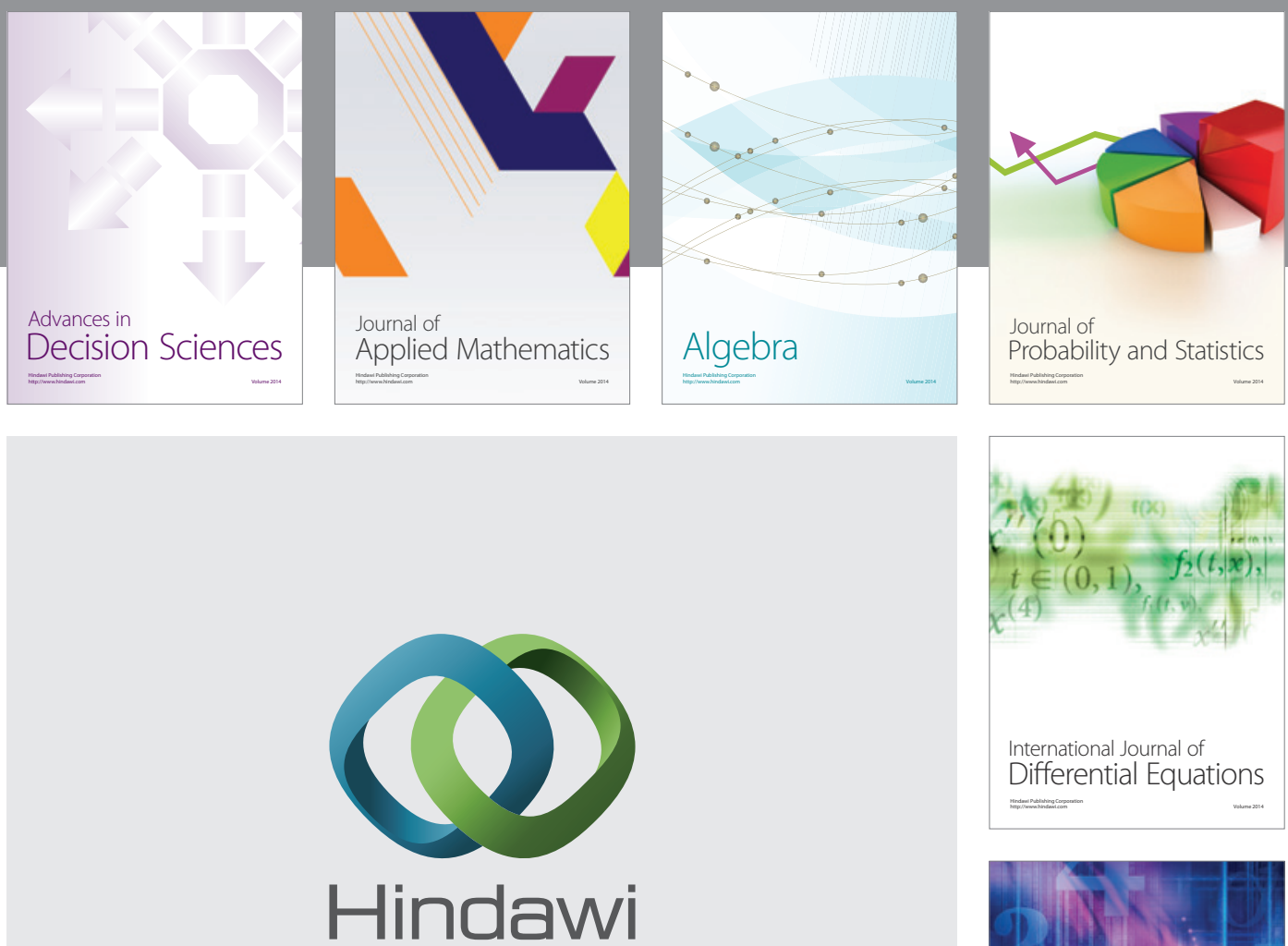

Submit your manuscripts at http://www.hindawi.com
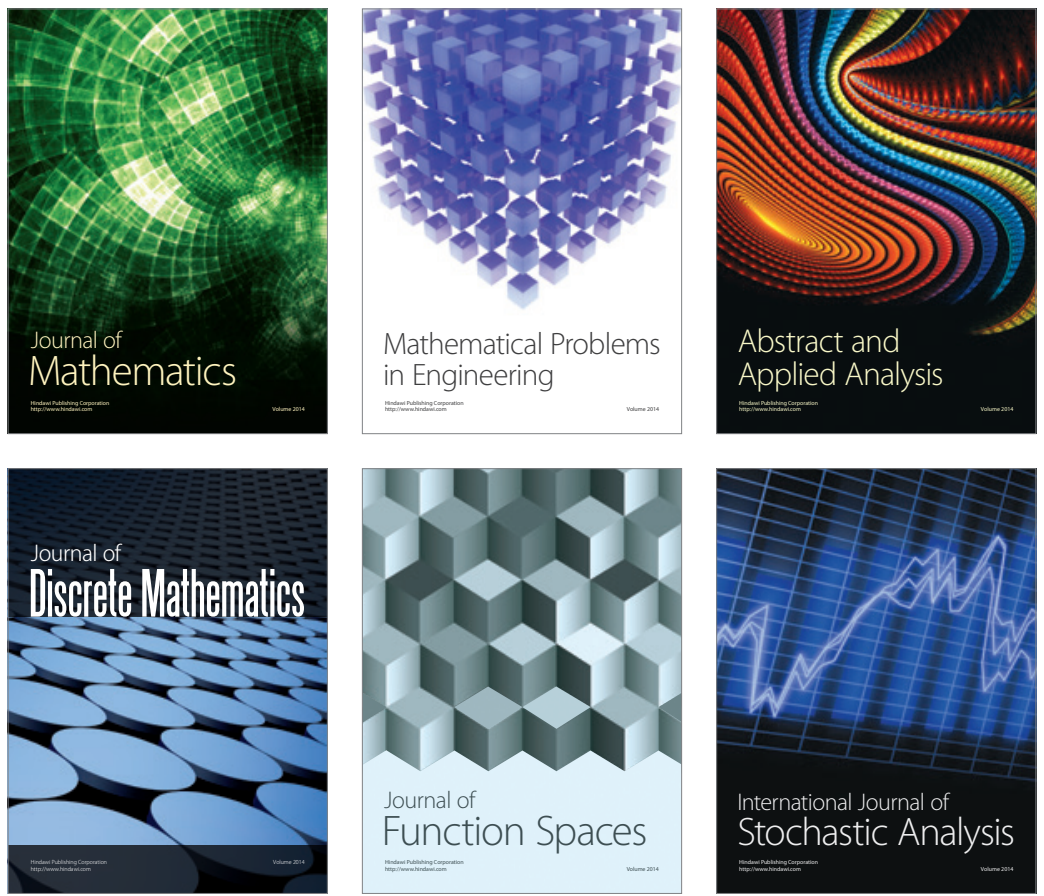

Journal of

Function Spaces

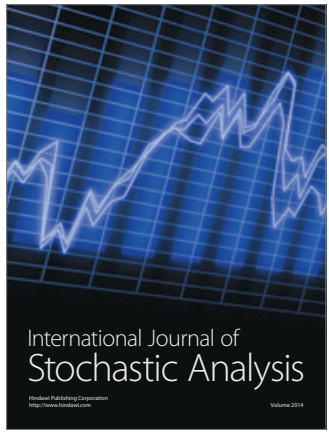

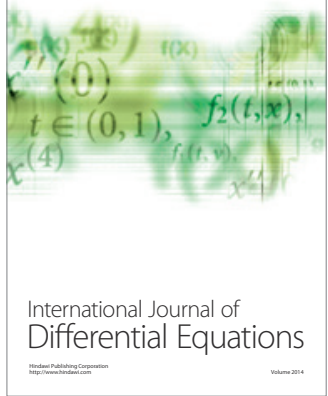
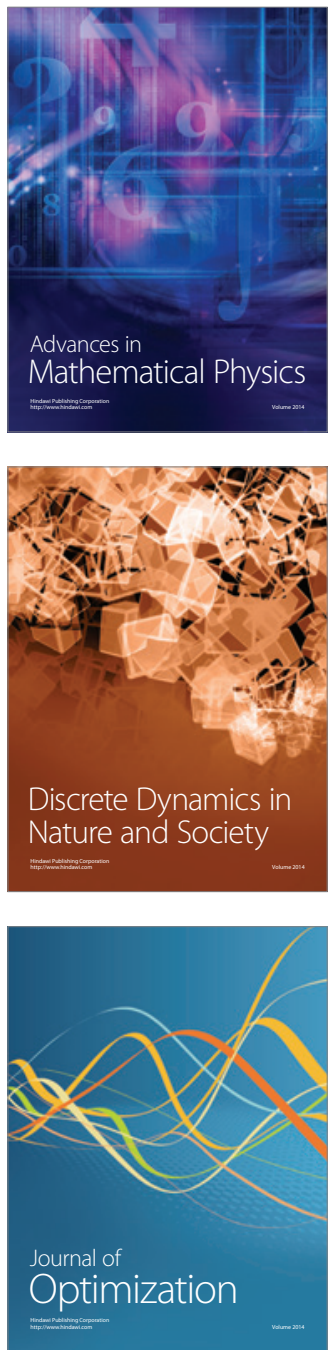